\title{
A new energy-aware wireless sensor network evolution model based on complex network
}

\author{
Shujuan Cui ${ }^{*}$ (D), Yuan Cao, Gengxin Sun and Sheng Bin
}

\begin{abstract}
For solving the problem of heterogeneous node energy and low energy efficiency in wireless sensor networks (WSNs), an effective and dynamic self-organization evolution model by introducing a complex network model is proposed in this paper. In the process of the evolution of WSN topology, it is considered that the nodes and links in the network are dynamic behaviors with increasing and decreasing, and combined this behavior with energy-awareness mechanism. The preferred attached mechanism and the anti-preferred deleted mechanism is closely related to the remaining node energy. The statistical characteristics of this network model are analyzed by theoretical calculation and mathematical simulation under different energy distribution functions. Through the simulation experiment, it can be proven that the proposed evolution model will cause the energy consumption of the whole wireless sensor network to be more balanced; at the same time, it would have an excellent performance of random node error tolerance.
\end{abstract}

Keywords: Wireless sensor networks, Complex network, Energy-balanced, Evolution model, Performance optimization

\section{Introduction}

Since the end of the twentieth century, the discovery of small-world network characteristics and scale-free network characteristics has aroused the interest of researchers to explore various evolving mechanisms of complex networks and dynamic behaviors [1]. Complex network theory has become an important analytical tool and research method in many fields. The complex network theory aims to study the large-scale network in the objective real world based on the viewpoint of systematic science, such as the international Internet, e-mail network, power network [2], aviation network, food chain network, metabolism network, research cooperation network [3], language network, social network [4], protein network [5], World Wide Web [6], software engineering network, and wireless network [7]. Any typical complex network can be abstracted as the connection between thousands of nodes and edges between two nodes, where nodes represent individuals (objects) in an actual complex network system, while edges represent relationships between individuals (objects).

\footnotetext{
* Correspondence: sungxmail@126.com

School of Data Science and Software Engineering, Qingdao University, Qingdao 266071, China
}

Small-world network model and scale-free network model provide a new perspective for the research of the real complex network system, but these two basic models still show some limitations in simulating the complex network in the real world. For example, in the world trade relations network, a node in the network means a country; the existence of edges between the two nodes indicates the existence of trade relations between the two countries. The evolution rule of complex networks in the actual world will be affected by many different complex factors.

Due to its complexity and dynamics, the wireless sensor network (WSN) can be used to study its inherent characteristics by complex network theory. The topology of network has important influence on WSN in the term of the energy efficiency and data transmission performance. The current classic model of network is different from the actual WSN. The complex network theory provides a new method to build a robust and efficient dynamic topology of WSN. Many researchers have used complex network theory to analyze the topology of WSN, for example. The reliability of network and the capacity of nodes are considered, and the dynamic evolution of wireless network is analyzed. The change of 
power law index is considered. A random deployment model is proposed, and the degree of the node evolves with the power law of $k^{2 /(\alpha-1)}$.

Although the scale-free model depicts the two basic principles of "growth" and "preferential" connection of power law distribution, it still has some limitations. Researchers explored the more complex formation mechanism and actual characteristics in the real system and established a related dynamic network evolution model to simulate the self-organization evolution process of real networks and set up a more accurate network evolution model for the complex networks of different types of real world.

There had been a lot of topological structure evolution models based on energy balance and energy awareness for WSNs in the past years. In WSNs, the node energy consumption directly affects the life cycle of WSNs. Mohite [8] further proposed the combinatorial evolution model based on the scale-free model. Huang et al. [9] proposed a new evolving model of networks and pointed out that, in a certain parameter range, the network generated by the new model still has the distribution characteristics of power law distribution. The nonlinear priority connection strategy is considered. Kumar and Ahlawat [10] proposed a new evolving model of networks, which used the node degree parameter with power index $(0 \sim 1)$ to replace the linear relationship in the BA scale-free network model. Wang et al. [11] proposed a new model by selecting the superior and eliminating the inferior mechanism. Miyao et al. [12] proposed a weighted evolving model of networks. Gao and Yang [13] proposed a competitive weighted evolving model of networks, which introduced the mechanism of the fittest rich. However, almost all existing studies do not research the energy efficiency for wireless sensor networks from the angle of complex network topology evolution and degree distribution.

Based on the above analysis, a new topology selforganization evolution model based on energy awareness for WSNs is proposed. And the dynamic behaviors of nodes and links in WSNs are considered. On the one hand, it reflects the evolution of sensor network. On the other hand, it can optimize the topology structure of WSN by adjusting some parameters. By means of the research method of complex network theory, the topology characteristics of WSNs are introduced into the dynamic evolution mechanism of complex network. The topology evolution of WSNs is analyzed in depth; the dynamic evolution in WSN closely related to the node energy is considered.

The method is given in Section 2. We propose the evolution model based on energy awareness of node and energy balance throughout the network in Section 3. In Section 4, the simulation analysis is given to verify the topological features of the network generated by the proposed model. At last, conclusion is given in Section 5 .

\section{Method}

Node energy is an important factor. It has an effect on the topology of WSN. In the network, the node energy is different from each other. It is very important to study how to consume energy more efficiently in the network environment in which the node energy is heterogeneous and extends the life cycle of sensor network. There are many studies that have been conducted in the network evolution of energy factors, for example, consider the heterogeneous characteristics of the network, a topology model of WSN cluster based on random walk is proposed by applying the complex network evolving theory. Other researchers analyzed the topology control of WSNs from the perspective of network evolution. The effectiveness of the energy consumption of WSN node on the basis of pure growth characteristics of complex networks is considered. The researchers believed that the probability of choosing the new link is not only related to the degree of the node, but also related to the residual energy of the node. But these documents are only considered the evolution of pure growth in sensor networks; they do not consider the dynamic behavior of nodes and links in WSNs.

Many complex networks in the real world can be described by evolutionary models. As a typical complex network, WSN has its own unique characteristics. Due to the finite energy and power of the sensor nodes, the sensor nodes only communicate with their neighbor node, which shows that the evolution of the wireless sensor network conforms to the concept of local-word connectivity.

A new topology evolution model by selecting the superior and eliminating the inferior mechanism based on energy awareness for WSNs is proposed and studied. According to the reality of the WSN, by using mean-field theory, a scale-free network model is derived. Among them, the new node selected in the new link should meet the optimal probability of node energy, while the old node selected in the deleted link should meet the anti-choice probability of the node energy. A simulation experiment is designed. By analyzing the complex network characteristic parameters of sensor network, the validity of the model is verified. We proposed our energy-balanced technique and evaluated by simulation using R studio software. Simulation results were obtained in the same experimental conditions.

\section{Evolution model of WSNs for energy balance}

WSNs belong to the category of complex networks but at the same time have significant characteristics. On the one hand, sensor node energy is a very valuable resource in WSNs. In order to save node communication energy consumption, nodes often do not work at the maximum transmission power and usually use a multi-hop communication mode to send data. Therefore, in the design of the sensor network topology, the energy consumption 
rate of the node energy heterogeneous environment must be taken into consideration to extend the life cycle of the entire network. On the other hand, the topology of wireless sensor networks is a dynamically changing process. This paper considers from the following two perspectives: (1) new nodes and new links are added and (2) node due to depletion of node energy and link failure. Therefore, this paper further discusses the accurate modeling of sensor networks based on the scale-free BA network model and proposes that not only the new node and the new link are added to the approximate BA network model but also the old node and the old link are deleted.

The BA model is a random network model which is used to demonstrate a preferential attachment or a "rich-get-richer" effect. In the model, an edge is most likely to attach to nodes with higher degrees.

The network model shows a dynamic evolution process with increase and decrease. Combined with the D. Shi model, the characteristics of the WSN itself, namely, the preferential growth and anti-preferential deletion in the topology evolution of the WSN, are closely related to the residual energy of the node. The effectiveness of node energy consumption in WSNs is considered, and a topology evolution model by selecting the superior and eliminating the inferior mechanism is proposed.

For clearance, we list the main parameters of the proposed evolution model (Table 1).

The network initially has $n_{0}$ sink nodes, $m_{0}$ common nodes, and $e_{0}$ edges, and the network evolves at every time step $t$ according to following algorithm:

(1) Growth: Adding a new node at every time step $t$ is repeated, and $m$ existing old nodes are connected. When choosing to establish a link with an existing old node, new joining node adopts preferred preference probability. Assume that the probability of a node establishing an edge with an old node $i$ is $\Pi\left(k_{i}\right) ; k_{i}$ represents the degree of node, and $E$ represents current remaining energy of node. In this paper, we define a function $f(E)$ to express the relationship between remaining energy of node

Table 1 Main parameters of the model

\begin{tabular}{ll}
\hline Parameters & Definition in evolution model \\
\hline$M_{0}$ & Initial amount of sensors \\
$n_{0}$ & Initial amount of sink sensors \\
$m$ & Edges were deleted per time step \\
$k_{i}$ & Degree of node $i$ \\
$E$ & Residual energy of each node \\
$\prod_{i} k_{i}$ & Probability of a new node \\
$\beta$ & An adjustment factor \\
$f(E)$ & Amount of sensors in each new adding sensor's range \\
$t_{i}$ & Time of sensor $i$ adding into WSN \\
\hline
\end{tabular}

and relationship between these nodes selected to establish a connection with the newly added node. The following results are obtained:

$$
\prod\left(k_{i}\right)=\frac{f(E) k_{i}}{\sum_{j} f\left(E_{j}\right) k_{j}}
$$

In practical applications of WSNs, the more energy the remaining node has connected to other nodes, the more energy will be consumed per unit of time. After a period of time, all nodes in the entire network reach equilibrium, and the network's life cycle is extended. This rule is followed in this chapter; the greater the node energy, the more likely it is to connect to the newly added node. Then, we introduce that $f(E)$ is an increasing function with the arguments $E$, such as $\beta \mathrm{E}_{\mathrm{i}}, \beta \mathrm{E}_{\mathrm{i}}^{2}$, where $\beta$ is an adjustment factor. General principles are taken into account, assuming $\beta=1$ and $f\left(E_{\mathrm{i}}\right)=\mathrm{E}_{\mathrm{i}}$, then the probability $\Pi\left(\mathrm{k}_{\mathrm{i}}\right)$ that the new node selection is linked to the old node $i$ is subject to the following rules:

$$
\prod\left(k_{i}\right)=\frac{E_{i} k_{i}}{\sum_{j} E_{j} k_{j}}
$$

(2) Delete link: If $m$ links are deleted with probability $p$ at every time step $t$, the $m p$ link was deleted. First, the anti-primary probability $\Pi^{*}\left(\mathrm{k}_{\mathrm{i}}\right)$ is used in the node with at least one link. That is, the smaller the remaining energy of sensor node, the probability of being selected to delete the link is more.

$$
\prod^{*}\left(k_{i}\right)=\frac{\left(k_{i} E_{i}\right)^{-1}}{\sum_{i}\left(k_{i} E_{i}\right)^{-1}}
$$

Secondly, for those nodes connected with node $i$, node $j$ would be selected with anti-preferential probability. That is, the node $j$ is selected from the neighborhood $\left(\mathrm{O}_{\mathrm{i}}\right)$ of node $i$ (o.r) with probability $\mathrm{K}_{\mathrm{i}}^{-1} \prod^{*}\left(\mathrm{k}_{\mathrm{j}}\right)$, where $\mathrm{K}_{\mathrm{i}}=\sum_{\mathrm{j} \in \mathrm{O}_{\mathrm{i}}}$ $\Pi^{*}\left(\mathrm{k}_{\mathrm{j}}\right)$. Then, delete the links of nodes $i$ and $j$ and repeat this step $m p$ times. The anti-preferential deletion is consistent with the topological dynamic changes caused by node and link failure in WSN. When remaining energy of node in the network is consumed to a certain threshold after a period of time, the link with which it is connected will fail and be deleted. The anti-preferred deletion mechanism exists in many practical networks. For example, in a user's e-mail network, a user's address book is used as a node, and an address in the address book is used as a link. When the address in the address book has not been used for a long time, the link represented by this address will be deleted in the e-mail network that has evolved. Similarly, in the language network, when some old expressions and sentences are no longer used, some languages will be isolated and deleted. In this paper, isolated nodes with a 
degree of 0 will also be permanently deleted from the network.

According to the continuous field theory, $k_{\mathrm{i}}(t)$ meets the demand of following dynamic formula:

$$
\begin{aligned}
\frac{\partial k_{i}}{\partial t} & =m \prod\left(k_{i}\right) \\
& =m p\left[\prod^{*} k_{i}+\sum_{j \in \operatorname{linked}(i)} \prod^{*}\left(k_{j}\right) K_{i}^{-1} \prod^{*}\left(k_{j}\right)\right]
\end{aligned}
$$

The first item in the formula expresses the link added by the preferred connection of the newly added node; the second item expresses the link that has been reduced by anti-preferred deletion. By using the mean field theory, the following formula can be introduced:

$$
\sum_{j} E_{j} k_{j}=N(t) * \bar{E} *\langle k(\mathrm{t})\rangle
$$

where $N(t)$ represents the amount of nodes in WSN which evolved to the time step $t . \overline{\mathrm{E}}$ represents the expectation of node energy in the network. $\langle k(t)\rangle$ represents average degree of sensor node at the time step $t$.

Assuming that sensor network has evolved over a longer time step to obtain a larger scale, $N(t)-1=\mathrm{n}_{0}+\mathrm{t} \approx \mathrm{t}$, the following formula can be obtained:

$$
\langle k\rangle=\frac{2 m(1-p) t+e_{0}}{n_{0}+t} \approx 2 m(1-p)
$$

And because $\sum_{\mathrm{j} \in \mathrm{O}(\mathrm{i})} \mathrm{K}_{\mathrm{i}}^{-1} \prod^{*}\left(\mathrm{k}_{\mathrm{i}}\right) \approx 1$, at each time step, $\Pi^{\prime \prime}\left(\mathrm{k}_{\mathrm{i}}\right) \approx 1 / \mathrm{N}(\mathrm{t}) \approx 1 / \mathrm{t}$, the following formula can be obtained:

$$
\frac{\partial k_{i}}{\partial t} \approx m \frac{E_{i} k_{i}}{2 m(1-p) \bar{E} t}-\frac{2 m p}{t}
$$

Obviously, $0 \leq p<1$. When $p=1$, the network no longer grows; this situation does not apply to the evolution of the sensor network and will not be analyzed here. For further analysis of the impact of the deletion probability $p$ on the network evolution, this paper discusses two cases of $p=0$ and $0<p<1$.

Case A: $p=0$

In this case, there is only a simple growth process of nodes and links in the network evolution process, and there is no deletion of nodes and links. This situation is suitable when the nodes in the WSN are in full energy, and in the ideal wireless data transmission and receiving environment, the topology discovery stage, the network simply grows without node and link deletion. Then, $k_{i}(t)$ satisfied

$$
\frac{\partial k_{i}}{\partial t}=m \frac{E_{i} k_{i}}{N \bar{E}\langle k(t)\rangle}=\frac{E_{i} k_{i}}{2\left(n_{0}+t\right) \bar{E}} \approx \frac{E_{i} k_{i}}{2 t \bar{E}}
$$

According to $k_{i}\left(t_{i}\right)=m$, the following formula can be gotten

$$
k(\mathrm{i}, \mathrm{t})=m\left(\frac{t}{i}\right)^{\frac{E_{i}}{2 \bar{E}}}
$$

The probability which a sensor node satisfies $k_{i}(t)<k$ at time step $t$ is:

$$
\mathrm{P}\left(k_{i}(\mathrm{t})<k\right)=\mathrm{P}\left(t_{i}>\left(\frac{m}{k}\right)^{2 \bar{E} / E_{i}} t\right)
$$

In this paper, one of the most common types of node addition methods in engineering, namely equal time intervals, is considered. Therefore, $t_{i}$ has an equal probability density $P\left(t_{i}\right)=1 / n_{0}+t$; substituting Eq. (7) can get

$$
\mathrm{P}\left(k_{i}(\mathrm{t})<k\right)=1-\left(\frac{m}{k}\right)^{2 \bar{E} / E_{i}} \frac{t}{n_{0}+t}
$$

The node probability density function with energy $E$

$$
\mathrm{P}\left(k_{E}\right)=\frac{\partial P\left(k_{i}(t)<k\right)}{\partial k}=\frac{2 m^{2 \bar{E} / E_{i}} t}{n_{0}+t} \frac{1}{k^{2 \bar{E} / E_{i}+1}}
$$

The overall degree distribution probability density function is

$$
\begin{aligned}
& P(k)=\int_{E_{\min }}^{E_{\max }} \rho(E) P\left(k_{E}\right) d E=\int_{E_{\min }}^{E_{\max }} \rho(E) \frac{2 m^{2 \bar{E} / E_{i}} t}{n_{0}+t} \frac{1}{k^{2 \bar{E} / E_{i}+1}} d E \\
& \propto k^{-\gamma} \int_{E_{\min }}^{E_{\max }} \rho(E) \frac{2 m^{2 \bar{E} / E_{i}} t}{n_{0}+t} d E \quad t \rightarrow \infty
\end{aligned}
$$

where, $\rho(E)$ represents the probability density distribution of node energy in the entire network, $E_{\min }$ and $E_{\max }$ represent their minimum and maximum values, respectively, and $\gamma$ represents a power law index. The network degree distribution shows a power-law formless scale characteristic.

Case B: $0<\mathrm{p}<1$

In this case, the general situation of the WSN is considered. In the evolution process, the network is not simply growing. That is, there is not only the increase of links and nodes, but also the deletion of nodes and links.

Equation (1) can be written as follows:

$$
\frac{\partial k_{i}}{\partial t}=m \frac{E_{i} k_{i}}{2 m(1-p) \bar{E} t}-\frac{2 m p}{t}
$$

The first-order linear partial differential equation is solved; according to the initial condition $k_{i}\left(t_{i}\right)=m$, the solution to Eq. (1) is

$$
k_{i}(t)=B\left(\frac{t}{t_{i}}\right)^{\beta} \quad \text { for large } t
$$

where $\beta$ represents the dynamic index and the value of $\beta$ is 


$$
\beta=\beta(m, p)=\frac{m E_{i}}{[2 m(1-p)+1] \bar{E}}
$$

The coefficient $B$ is

$$
B=B(m, p)=m-\frac{m-2 m p[2 m(1-p)+1]}{m} \frac{\bar{E}}{E}
$$

We can get the probability that $k_{i}(t)$ is less than $k$ :

$$
P\left(k_{i}(t)<k\right)=P\left(t_{i}>\left(\frac{B}{B-m+k}\right)^{1 / \beta} t\right) \quad \text { for } k>m
$$

In this paper, the most common way to increase the number of nodes in a project is considered. Assume that the nodes join the WSN at equal time steps; therefore, $t_{i}$ has an equal probability density $P\left(t_{i}\right)=1 / n_{0}+t$; we can draw the conclusion

$$
P\left(k_{i}(\mathrm{t})<k\right)=1-\left(\frac{B}{B-m+k}\right)^{1 / \beta} \frac{t}{n_{0}+t}
$$

The node probability density function with energy $E$ is

$$
\begin{aligned}
P\left(k_{E}\right) & =\frac{\partial P\left(k_{i}(t)<k\right)}{\partial k} \\
& =\frac{t}{n_{0}+t} \frac{1}{\beta} B^{1 / \beta}(k+B-m)^{-\left(1+\frac{1}{\beta}\right)}
\end{aligned}
$$

The probability density function of the network is

$$
\begin{aligned}
P(\mathrm{k}) & =\int_{E_{\min }}^{E_{\max }} \rho(E) P\left(k_{E}\right) d E \\
& =\int_{E_{\min }}^{E_{\max }} \rho(E) \frac{t}{n_{0}+t} \frac{1}{\beta} B^{1 / \beta}(k+B-m)^{-\left(1+\frac{1}{\beta}\right)} d E
\end{aligned}
$$

where, $\rho(E), E_{\min }$, and $E_{\max }$ are defined in the same way as in Eq. (14). $\rho(E)$ is the probability density distribution of node energy in the whole network. $E_{\min }$ and $E_{\max }$ are their minimum and maximum values, respectively. It can be seen that the degree distribution index is closely related to [ $E_{\min }, E_{\max }$ ] and the deletion probabilities $p$ and $E_{\mathrm{i}}$, but not to the node energy distribution.

\section{Simulation and discussions}

For verifying the effectiveness of the proposed network evolution model, some simulation experiments were performed. By simulating and analyzing various statistical characteristics of topology, the complex dynamics of the proposed model can be better understood, and the influence of node energy distribution function $\rho(E)$ on topology structure can be deeply understood. In this section, the simulation results of various statistical parameters such as the degree of network topology distribution, the relationship between the node degree and energy, the correlation degree, and the network energy efficiency, under different evolution parameters will be given. These characteristics are the statistical feature parameters that researchers pay attention to in their studies of complex network models and also important indicators for evaluating the performance of sensor networks.

In the simulation experiment in this paper, we used the energy distribution of the nodes in the network to be between 0.6 and $1 \mathrm{~J}$ and considered the following three different node energy distribution functions $\rho(E)$ : (1) all nodes in the network have the same energy, all of them are $0.5 \mathrm{~J}$, and their energy value variance is 0 ; (2) uniform distribution and their energy variance is $1 / 12$; and (3) exponential distribution $(\exp (-E))$, its variance is 1. According to the physical meaning of the variance in probability statistics, the variance value can be used to indicate the heterogeneity of node energy. The larger the variance, the greater the heterogeneity of the energy in the nodes are. In the above three cases, the node energy distribution with exponential distribution in the third case has the greatest heterogeneity. In the first case, the node energy has isomorphism.

The distribution functions and corresponding mathematical expectation of remaining energy are shown in Table 2.

The degree distribution is considered as the most important factor to distinguish the characteristics of real networks. In order to calculate its value, an empirical research method was adopted. The evolution model by selecting the superior and eliminating the inferior mechanism was used. Through programming, specific network models are generated. Then, the frequency of the node with degree $k$ is counted, repeated several times, and its average value is taken, and then, an approximate value of the probability $P(k)$ is represented by the frequency, that is, the proportion of the amount of nodes whose degree is $k$ is probability $P$. The influence of energy distribution of sensor nodes is shown as Fig. 1. With various degree distributions, various mathematical expectations will get different growing speeds.

From Fig. 2 and Fig. 3, it can be known that the relationships are between the range of adding node and the degree distribution.

Figure 2 shows that the coming sensors will choose different quantity of nodes based on the scope. It can be

Table 2 Various distributions of residual energy

\begin{tabular}{ll}
\hline Distribution of residual energy $R$ & $\begin{array}{l}\text { The mathematical expectation } \\
\text { of residual energy }\end{array}$ \\
\hline$\rho(E)=2$ & 0.75 \\
$\rho(E) \sim E-1$ & 0.6 \\
$\rho(E) \sim E-2$ & 1 \\
\hline
\end{tabular}




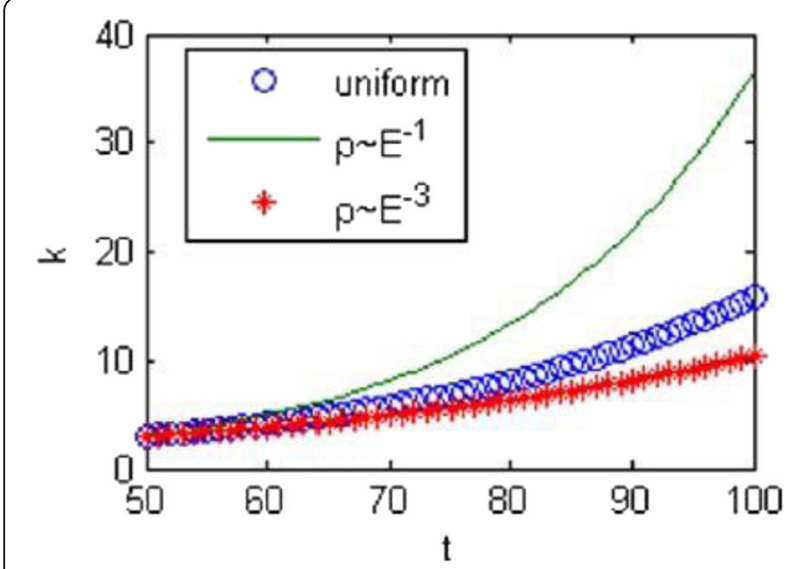

Fig. 1 Degree of evolution network with various energy distributions

seen that the more the $S$ is small, the more the probability of sensor $i$ connected is big.

For a clearer illustration of the influence of the node energy distribution function on the internal connection of the network and to reveal the complexity of the internal connection of the network topology, the correlation between the node degree and its energy and the degree correlation are simulated and analyzed. Figure 4 shows the influence of a new node on the connectivity growth. It can be seen that the elder nodes had more possibility of being chosen for connecting to the coming nodes; it implies that connectivity of the elder will increase faster than those of new coming.

The degree distributions generated by the proposed model with various energy distributions are shown in Fig. 4.

From Fig. 4, we can see that the degree distribution follows power law. It implies that scale-free wireless sensor networks in any condition all can be gotten by our evolution model. So we do not need to consider the residual energy while constructing networks.

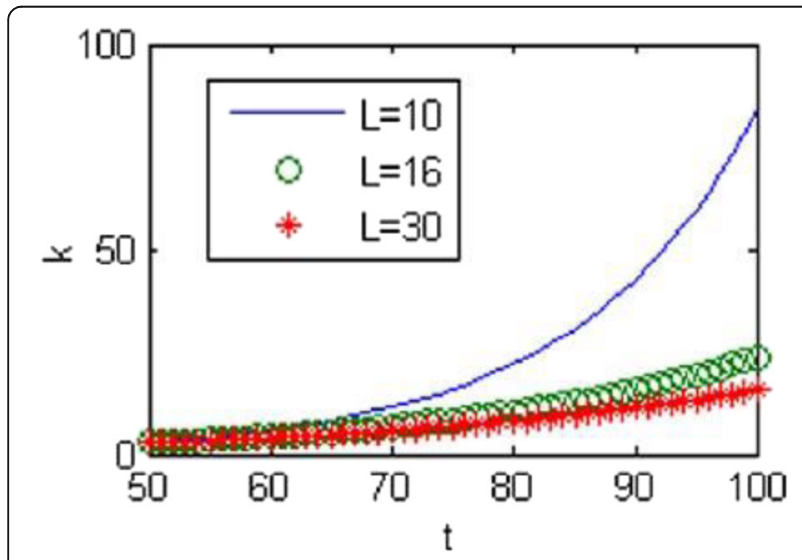

Fig. 2 Degree of evolution network with different local-scope

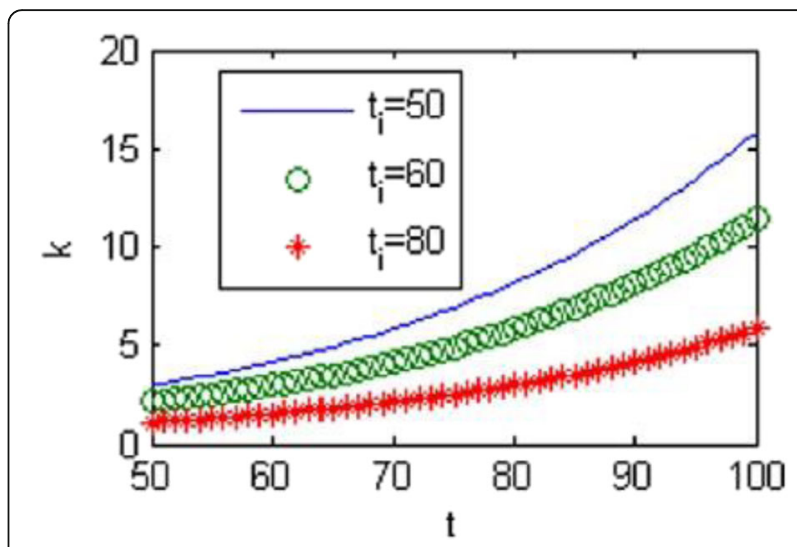

Fig. 3 Degree of evolution network with various node incoming time

In experiments, we do not take the limitation of max connectivity $k_{i}$ into consideration; when actual influence factors are put into the modified model, the degree distribution with different energy distributions are shown individually in Fig. 5 and Fig. 6.

Figure 5 shows that degree distribution of networks which are generated from the modified model when the residual energy distribution is unifying. Those sensors with large connectivity would raise their degree slowly because of the limitation of the max degree. Our modified model prevents the sensors from consuming their power, and then, the energy of the overall network would be balanced.

Characteristic path length and network energy efficiency are usually used to analyze the ability to communicate between nodes in a network. These two indicators are defined. Small feature path lengths and large network energy efficiency mean that fewer hops are required for data transmission. Energy consumption for data transmission

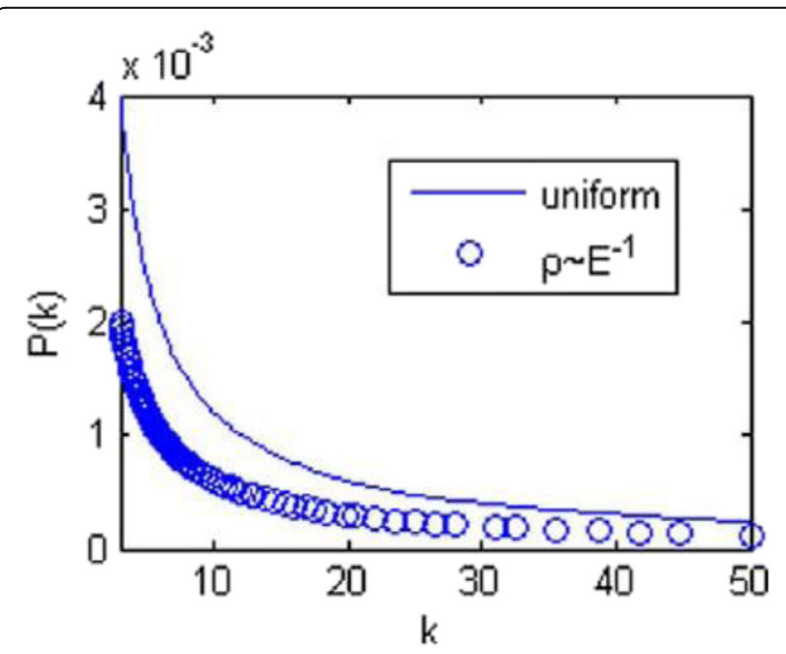

Fig. 4 The degree distribution with various energy distributions 


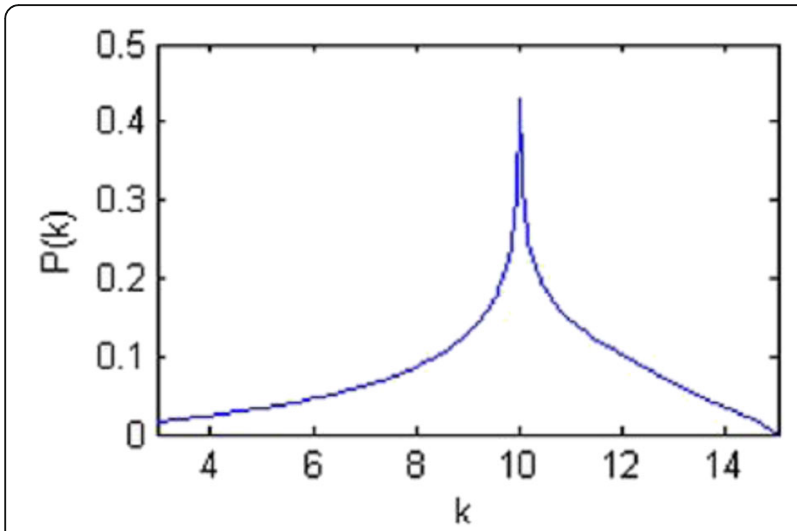

Fig. 5 The degree distribution with uniform energy distributions

is saved. The comparison of connectivity evolution with various sizes of scope is shown as Fig. 6 .

The connectivity evolution by various time of a sensor added to the network is shown in Fig. 7. From Fig. 6 and Fig. 7, it can be seen that the increment of the connectivity is much slower because of the max connectivity limitations.

\section{Conclusions}

This paper firstly introduced the origin and development history of small-world networks and then analyzed the application and research of small-world effects in wireless sensor networks. The sensor network is a dynamic network with a constantly changing topology. Based on the study on the evolution model by selecting the superior and eliminating the inferior mechanism, the energy perception mechanism is introduced, combined with the fact that the preferential growth and anti-preferential were deleted in the WSN topology which are closely related to the residual energy of the node. A new topology evolution model by selecting the superior and eliminating the inferior mechanism based on energy awareness for WSNs is

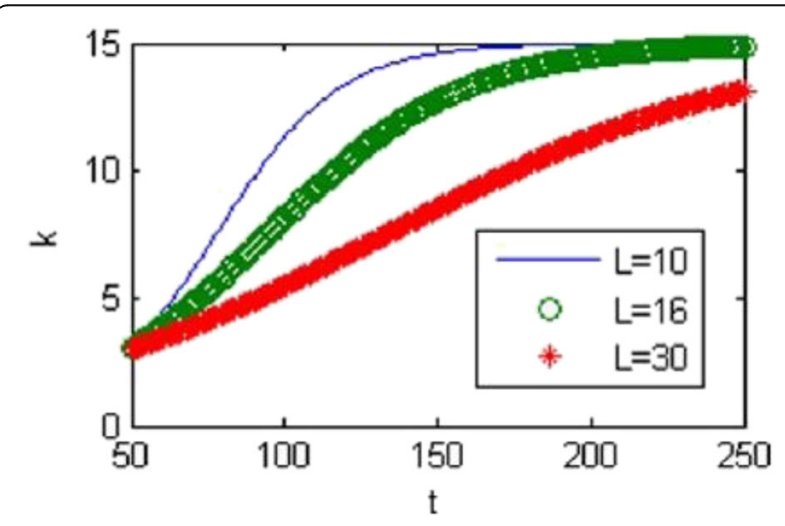

Fig. 6 Degree of network with different local-scope by modified model

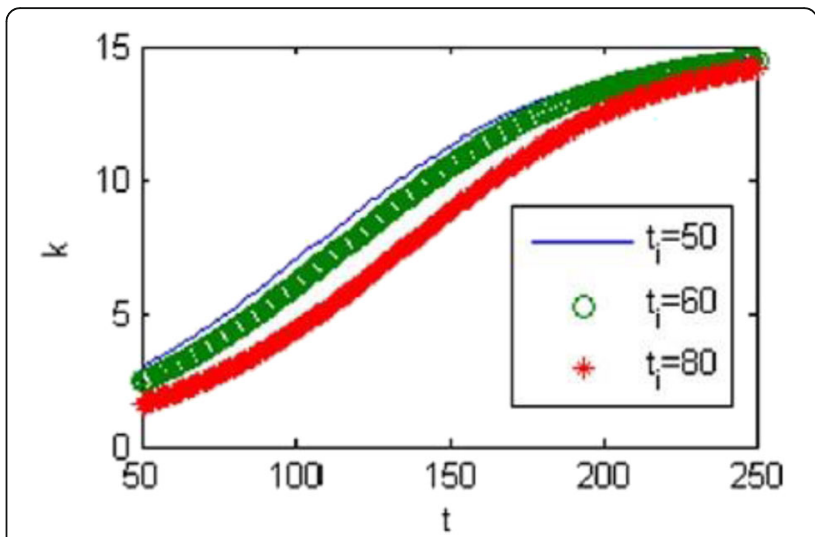

Fig. 7 Degree of network with different $t_{i}$ by modified model

proposed. The model takes into account the evolution process of wireless sensor networks considering the node energy-aware priority connection mechanism and the node and link of the sensor network. Through simulation results, it was verified that the proposed model can well reflect the evolution of wireless sensor networks under different node energy distribution scenarios.

Abbreviations

WSANs: Wireless sensor and actuator networks; WSNs: Wireless sensor networks

\section{Funding}

This work is supported by the Shandong Provincial Natural Science Foundation, China, under Grant No. ZR2017MG011.

\section{Authors' contributions}

SJC, YC, and GXS contributed to the conception and evolution model of the study. GXS and SB contributed to the acquisition of simulation. SJC, GXS contributed to the analysis of simulation data. All authors read and approved the final manuscript

\section{Author's information}

Shujuan Cui received a M.S. degree from Qingdao University, in 2004. She is an assistant professor in Qingdao University. Her research interests lie in wireless sensor networks (e-mail: sungxmail@126.com).

Gengxin Sun received a Ph.D. degree from Qingdao University, in 2013. He is an associate professor in Qingdao University, China. His research interests lie in complex network (e-mail: sungengxin@qdu.edu.cn).

Sheng Bin received a Ph.D. degree from Shandong University of Science and Technology, in 2014. She is an associate professor in Qingdao University, China.

Her research interests lie in complex network (e-mail: binsheng@qdu.edu.cn).

\section{Competing interests}

The authors declare that they have no competing interests.

\section{Publisher's Note}

Springer Nature remains neutral with regard to jurisdictional claims in published maps and institutional affiliations.

Received: 12 June 2018 Accepted: 30 August 2018

Published online: 10 September 2018

\section{References}

1. C. Zhang, Cluster-based routing algorithms using spatial data correlation for wireless sensor networks. J. Commun. 5(3), 232-238 (2010). https://doi.org/ 10.4304/jcm.5.3.232-238 
2. J. Wang, J. Kim, L. Shu, A distance-based energy aware routing algorithm for wireless sensor networks. Sensors. 10(10), 9493-9511 (2010). https://doi.org/ 10.3390/s101009493

3. S. Cai, P. Zhou, Z. Liu, Synchronization analysis of directed complex networks with time-delayed dynamical nodes and impulsive effects. Nonlinear. Dynamics. 76(3), 1677-1691 (2014). https://doi.org/10.1007/ s11071-014-1238-z

4. G. Sun, S. Bin, Router-level internet topology evolution model based on multi-subnet composited complex network model. J. Internet. Technol. 18(6), 1275-1283 (2017). https://doi.org/10.6138/IT.2017.18.6.20140617.

5. N. Chua, K. Ning, D. Zueco, Using indirect protein-protein interactions for protein complex prediction. Comput. Syst. Bioinforma. 6(3), 97-109 (2011). https://doi.org/10.1142/9781860948732_0014.

6. G. Paparo, M. Müller, F. Comellas, Quantum Google in a complex network. Sci. Rep. 2773(3), 127-132 (2013). https://doi.org/10.1038/srep02773.

7. W. Berry, P. Chartier, Identifying influential edges in a directed network: big events, upsets and non-transitivity. J. Complex. Netw. 2(2), 87-109 (2012). https://doi.org/10.1093/comnet/cnt018.

8. P. Mohite, Adaptive data fusion for energy efficient routing in wireless sensor network. Int. J. Energy. Optim. Eng. 17(4), 1-17 (2015). https://doi. org/10.4018/ijeoe.2015010101.

9. P. Huang, J. Chen, C. Wang, Compressed sensing algorithm based on data fusion tree in wireless sensor networks. J. Electron. Inf. Technol. 36(10), 2364-2369 (2014). https://doi.org/10.3724/SP.J.1146.2013.01621.

10. L. Kumar, M. Ahlawat, Evolution techniques for reliability of wireless sensor network, international journal of advances in engineering. Sciences. 2(4), 77-87 (2012). https://doi.org/10.1109/CEC.2011.5949636.

11. Q. Wang, J. Chen, H. Fang, Fault-tolerant topology control algorithm for mobile robotic networks. Int. J. Control. Autom. Syst. 12(3), 582-589 (2014). https://doi.org/10.1007/s12555-013-0195-5

12. K. Miyao, H. Nakayama, N. Ansari, LTRT: an efficient and reliable topology control algorithm for ad-hoc networks. Wirel. Commun. IEEE. Trans. 8(12), 6050-6058 (2009). https://doi.org/10.1109/TWC.2009.12.090073

13. H. Gao, Z. Yang, A novel wireless sensor network evolution model based on energy-efficiency. Int. J. Online. Eng. 13(03), 4-16 (2017). https://doi.org/10. 3991/ijoe.v13i03.6855

\section{Submit your manuscript to a SpringerOpen ${ }^{\circ}$ journal and benefit from:}

- Convenient online submission

- Rigorous peer review

- Open access: articles freely available online

High visibility within the field

- Retaining the copyright to your article

Submit your next manuscript at $\boldsymbol{\nabla}$ springeropen.com 